\title{
Lutz Unterseher Bürgerliches Arbeitsrecht oder die Zerstörung der formalen Rationalität
}

I.

Franz Neumann übersteigt in der Analyse des Funktionswandels des bürgerlichen Rechts die Ebene der rein juristischen Vernunft, indem er in der ökonomischen Sphäre und im Bereich der Normen annähernd synchrone Bewegungen aufdeckt ${ }^{1}$. So ist das generelle Gesetz, das wesentlich bürgerliche Privatautonomie und damit Vertrags- und Gewerbefreiheit garantiert, im Konnex mit der Phase des Konkurrenzkapitalismus in zwei Aspekten unmitrelbar systembezogen: als kalkulierbare Rechtsfolgegarantie am Markt prinzipiell ähnlich starker Kontrahenten und in der verschleiernden Formel der $\rightarrow$ rule of law *, die eine von der Klasse der Produzenten monopolisierte Parlamentsherrschaft verbirgt. In England - nach früher Emanzipation des Bürgertums und seiner damit einhergehenden Fusion mir dem Adel - bewahre sich die herrschende Klasse noch lange Zeit das Privileg des formal-rationalen Rechts².

In Deutschland, wo das Bürgertum erst domestiziert und dann - mittlerweile in der imperialistischen Phase - zum Pakt mit Krone, Armee und Beamtenapparat selektiv zugelassen wird, erscheint die Herrschaft des generellen Gesetzes in der spezifischen Ausprägung des $*$ Rechtsstaates unter einem weiteren, nun nicht unmitrelbar systemorientierten Aspekt. Der $\gg$ Rechtsstaat* gilt für alle, garantiert die gleiche Anwendung der Gesetze, die gesetzesmäßige Verwaltung samt der an die Konstruktion der Rechtsperson geknüpften ökonomischen aber auch persönlichen und politischen Grundfreiheiten.

Damit erlangt das rationale Rechtssystem zumindest den Anschein, Vehikel einer Transzendenz des bestehenden Gesellschaftszustandes zu sein - und zwar in zweierlei Hinsicht: Auf spekulativer Ebene gewinnt es die Eigenschaft einer Mindestbedingung jeder lebenswerten konkreten Utopie; als Teil der historischen Realität wird es zum Rückhalt des kämpfenden Proletariats, zum formalen Versprechen, das nach inhaltlicher Einlösung verlangt. Aber eben diese formale Struktur impliziert mähliches, zögernd kalkulierendes Prozedieren der Ausgebeuteten und wird - zumal sie alsbald die kleinen Freuden der Organisation gewährt - schließlich zum Leitfaden der gesellschaftlichen Integration, die realiter durch die Waffenmacht des herrschenden Bündnisses abgesichert wird.

Der Rechtsstaat ist in Deutschland Produkt der dekretierten Kodifikationen aufgeklärter Monarchen. Hierbei steht den beauftragten bürgerlichen Kodifi-

1 Vgl. Franz Neumana, Der Funktionswandel des Gesetzes im Reche der bürgerlichen Gesellschat, Neuauflage in: F. Neumann, Demokratischer und autoritärer Staat - Studien zur politischen. Theorie, Frankfurd/Wien 1967, S. 3 I ff.

Die Darstellung der Entwidklung vom gesdlossenen positiven Rechtssystem zur Dominanz des Billigkeitsrechts ist weitgehend hieran orientiert.

2 Vgl. Max Weber, Rechtssoziologie in: Wirtschaft und Gesellschaft I. Halbband, Köln/Berlin 1964, S. 603 . 
katoren inhaltliche Neuschöpfung in der Regel nicht zu. Bleiben so die verordneten Gesetze etwas Vorzufindendes, bringen einzig deren rationale Systematisierung und Interpretation Nutzen für ein beschwerlich sich freischwimmendes Bürgertum. Rechtsphilosophie, das ist immer auch $Z$ weifel an der Legitimation von Satzungen, wird abgeschaff. Das Gesetz muß Dogma sein, um willkürliche Eingriffe des Staates in die bürgerliche Privatautonomie abzuwehren, und durch den Richter formalistisch angewendet werden. Ein System logischer Begriffe regiert die Wirklichkeit, die - passiven Charakters - von der Rechtsfortbildung abgeschnitren erscheint. Diese Begriffsjurisprudenz suspendiert die Dialek rik von Sein und Sollen, von Norm und Gesellschaft, zugunsten eines Diktates der Norm³. Damit ist die Schwäche eines Bürgertums bezeichnet, das, ohne politischen Einfluß auf das Recht zu haben, ihm Minimalgarantien für den eigenen ökonomischen Aufstieg abzugewinnen trachtet und es so in seiner Formalstruktur verselbständigt.

Noch als im wilhelminischen Deutschland der Jahrhundertwende die Industrie einem Trend zur Konzentration von buschfeuerartiger Geschwindigkeit unterliegt, und eine imperialistische Exportorientierung samt einer Annäherung des Großbürgertums an die dominierenden vorindustriellen Gruppen sich durchsetzt, erhalten sich die nun zur Herrschaft zugelassenen Teile des Bürgertums ihre Fixierung an die Segnungen des Rechtsstaates, der für sie zuallererst Vertragsund Gewerbefreiheit bedeutet.

Zwar meint der soziale Begriff der Vertragsfreiheit, etwa in der Theorie Adam Smith's den Austausch gleichwertiger Leistungen durch gleich starke Kontrahenten. Die formal juristische Abstraktion dieses Begriffs bedeuter jede mögliche Vertragsfreiheit und damit auch vertragliche Unterwerfung Schwacher - Produzenten wie Proletarier. Der Vertrag als Vehikel der Monopolisierung ist im Kaiserreich erwünscht.

Seine die Herrschaft des generellen Gesetzes zerstörende Dialekrik tritr noch nicht offen zutage. Billigkeitsrecht - der Begleirer ökonomischer Konzentration setzt sich nicht durch, es sei denn neben den Gesetzen, in industrieeigener Schiedsgerichtsbarkeit.

Das System des formal rationalen Rechts hat nämlich eine integrierende Funktion. Es ist $z$ war reaktionären Interessen nützlich, schütze aber darüber hinaus die wirtschaftlich Schwachen vor Willkür. Dieses Moment erfährt eine Steigerung durch ein Armenrecht von hoher Rationalität - initiiert durch Bismarck, der Verkörperung sozialkonservativ beeinflußter Staatsmacht. "Der bewußte $Z$ weeck der sozialkonservativen Aktion war es, den kollekriven aktiven Eingriff der Arbeiterschaft als Klasse abzubiegen ${ }^{4}$. In dieser Abwehrhaltung treffen sich quasiabsolutistisches Staatsinteresse und Bourgeoisie, für die kein Grund besteht, das ihre Teilhabe an der Macht stabilisierende Rechtssystem zu durchbrechen und damit dessen Sanktionsgewalt zu gefährden.

Krone, Armee, Bürokratie, Großbürgertum und Grundbesitz haben ein Interesse an der wortgetreuen Anwendung der Gesetze. Diese illiberale Koalition wird vom bürgerlichen Stand der Richter, der aber - bedinge durch seinen sozialen Ort - vorbürgerlichen Verhaltensweisen zuneigt, gegenüber dem Volk repräsentiert. Da der Staat mit Gesetzen herrscht, bedeutet Gesetzestreue Staatstreue. Die Interessen des Richterstandes werden so mit denen staatlicher Herr-

3 Vgl. Spiros Simitis, Die faktischen Vertragsverhälmisse, Ffm. I957, S. 6.

Die Lösung der Norm von der Gesellschatt hat durchaus eine gesellschaftliche Funksion! (L. U.).

4 Otto Kahn-Freund, Der Funktionswandel des Arbeitsrechts, in: Thilo Ramm (Herausgeber),

Arbeitsrecht und Politik, Neuwied 1966, S. 214. 
schaft verquickt, daß ihr Klassencharakter zumindest den Richtern selbst verborgen ist und deren in freiem Ermessen sich äußernde Kritik ausbleibr.

*Unabhängigkeit der Rechtspflege? de jure hat sie nie jemand angezweifelt, de facto nie jemand angestrebt ${ }^{3}$.

Der höhere Richter hatte als Reserveoffizier und Staatsanwalt gehorchen gelernt. Der Satz von der Unabsetzbarkeit und Unversetzbarkeit gilt nur sehr bedingt, d. h. für den ordentlichen Richrer. Assessoren, die, wie allgemein verbreitet, als Hilfsrichter Richteraufgaben erfüllen, können jederzeit abgesetzt oder versetzt werden. Die Assessorenzeit - nach vierjähriger Referendarzeit dauert durchschnittlich acht bis zehn Jahre. Diese unbesoldete Wartezeir fixiert die Schicht, aus der sich das Richtertum rekrutiert, als den gehobenen Mitrelstand. In der Regel können nur Söhne von Richtern und anderen höheren Beamten, deren Einkommen $z$ war nicht übermäßig hoch, aber auskömmlict und in seiner konstanten Höhe voraussehbar ist, eine derartige Ausbildung durchstehen. Schon in der Studienzeit kapselt sich der deutsche Jurist durch den Eintritt in eine Korporation von der übrigen Bevölkerung ab. $\gg \mathrm{Zu}$ dem Proletariat schlug keine soziale, gesellschaftiche oder geistige Brücke ${ }^{6}$.«

$I I$.

I 918 wird der früher ignorierte Konflikt $z$ wischen Arbeiterbewegung und Unternehmertum anerkannt und bald darauf durch die neue Verfassung institutionalisiert. Das Werkzeug ist der Vertrag, der nun nicht mehr - wie auch immer fingierte - Einzelpersonen in Beziehung setzt, sondern unverhülit gesellschaftliche Machtblöcke.

r. Ebert macht in einem Pakt mit der Obersten Heeresleitung (Hindenburg und Groener) die alte Armee zum Garanten der neuen Ordnung.

2. Mit Beschluß der Zentralarbeitsgemeinschaft (Stinnes-Legien) versprechen die Unternehmer, die gelben Gewerkschaften nicht mehr zu dulden, allein die unabhängigen Gewerkschaften anzuerkennen, mit ihnen zusammenzuarbeiten und die Arbeitsbedingungen durch Tarifvertrag zu regeln. Dieses Abkommen richtet sich nicht nur gegen den Bolschewismus, sondern macht jede Art der sozialistischen Umgestaltung unmöglich.

3. Im März I9r 9 vereinbart die SPD Berlins mit der Reichsregierung, Betriebsräte einzuführen und deren Verankerung in der Reichsverfassung. Diese Betriebsräte sollen mit den revolutionären Arbeiter- und Soldatenräten nichts zu tun haben.

4. Die Parteien von Weimar, Zentrum, SPD und Demokraten, einigen sich auf die Reetablierung der aiten Bürokratie und Justiz, auf die Sanktionierung bürgerlicher Freiheitsrechte, die nur leicht durch soziale Grundrechte eingeschränkt sind (Koalitionsf reiheit), und führen die parlamentarische Demokratie ein.

Der dieser politischen Neukonstruktion zugrundeliegende Kompromiß, der Pakt zwischen Kapital und Arbeit, trägt von vornherein den Keim seiner Zerstörung in sich. Die Macht des Proletariats ist durch seinen freien Zugang zum nun souveränen Parlament zwar gewachsen, doch hat es kein politisches Monopol. In der ökonomischen Sphäre jedoch sind die Strukturen des Kaiserreichs erhalten geblieben: Die Kontrolle der Produkrionsmittel liegt in den gleichen Händen, der

5 Ernst Fraenkel, Zur Soziologie der Klassenjustiz, Neuauflage, Darmstadt 1968, S. I2.

- Ernst Fraenkel, ג.2.O., S. I 3 . 
Prozeß der Konzentration beschleunigt sich weiterhin und die beschränkte ökonomische Macht der Arbeiterkoalitionen schwankt wie ehedem mit den Konjunkrurbedingungen, wovor auch die neuerlich formal verankerte Koalitionsfreiheit nicht bewahrr. An dieser Spannung zwischen Politik und Okonomie zerbrichr die Zentralarbeirsgemeinschaft.

Die Unternehmer werfen den Ballast an Parenerschaftsideologie über Bord, gehen den Weg des unverhüllten Klassenkampfes und der Einflußnahme auf ein meist bürgerlich kontrolliertes Parlament. Ihre pressure-groups arbeiten mit der Deutschen Volkspartei und zum Teil auch der Deutschnationalen Volkspartei zusammen. Die Labilität der im Reichstag sich bildenden Parteienbündnisse sowie die unbegründete Furcht vor einer Koalition aller politischen Vertreter des Proletariats verstärken das Interesse der industriellen Machthaber an einer gleichsam prophylaktischen Umgehung des Parlaments. Verwaltung und Justiz, von der Aufsicht der Legislative kaum berühr, werden zum freien Feld des außerparlamentarischen Einflusses gesellschaftlicher Machr.

Welche Rolle sucht sich der Richterstand in dieser Spannung $\mathrm{z} w i$ ischen politisch möglicher Macht des Proletariats und der etablierten gesellschaftlichen des Großkapitals? Die spezifische Weise seiner schichtgebundenen Rekrutierung ändert sich nicht wesentlich. Zumindest die Richter der höheren Instanzen entstammen durchweg einer Karriere im Kaiserreich. Jedoch die von Laband dem Monarchen zugeschriebene Sanktion der Gesetze, d. h. deren letzte Rechtfertigung, besteht plötzlich nicht mehr. Mit Fortfall der monarchischen Staatsspitze, die in der Vorstellung des Richtertums durch die Sanktion den Gesetzentwurf aus dem Interessenkampf und Parteienhader in eine parteilose, klassenfreie Sphäre erhoben hatte, sieht der Richter das Recht als Spielball der gesellschaftlichen $\mathrm{Kräfte}^{7}$.«

Durch die Geldentwertung fühlt die Richterschaft sich mit einem Male proletarisiert, oder doch in bedroblicher Nähe der Arbeiterschaft. Das verdeutlicht die Ignoranz der Richter gegenüber dem Status des Arbeiters, der etwa das Privileg auf Arbeitslosigkeit einschließt.

Ihren ökonomischen Niedergang versuchen sie durch enge Beziehungen zu sozial höherstehenden Gruppen zu kaschieren. Ressentiments gegen Neureiche haben, abgesehen von ihrem meist antisemitischen Hintergrund, eher den Charakter eines Freibriefs, die »wirklich * großen nationalen Wirtschaftsführer um so rüdkhaltloser bewundern zu können.

Die freilich zeitlich begrenzte wirtschaftliche Gefährdung des Richtertums und der voraufgegangene Verlust seines Bezugspunktes, der Krone, führen es zu einer Selbstbesinnung von der Art eines kleinbürgerlich-trotzigen Heroismus, zu einer Neuwertung seiner materiellen Standesinteressen.

Zur Grundlage dieser neuen Rolle wird seine Unabhängigkeit gemacht. Es gilt $\mathrm{ihm}$, diese als formale Hülle aus dem Kaiserreich herübergeretrete Garantie nun mit Inhalt anzufüllen. Die im Kaiserreich so geschmähten, weil oppositionellen freirechtlichen Gedanken gewinnen schlagartig an Aktualität. Der »königlichex Richter der Freirechtsschule, eine zahlreiche Reproduktion des abgeschfften Monarchen auf niedrigerer Ebene, steilt sich nun mit freiem Ermessen über das Gesetz, das ihm nur mehr Richtschnur, nicht Befehl ist. Mittels der elastischen Normen, die früher mehr den Charakter einer Randexistenz besaßen, besonders mit $\operatorname{dem} \ 242 \mathrm{BGB}$, werden andere inhaltlich bestimmte Normen verdrängt.

Vom Formalismus zum Finalismus: Nicht der Ursprung, der Zweck des Geset-

7 ebenda, S. 9. 
zes wird zum Schlüssel der Interpretation und damit zum Kriterium seiner An-

wendbarkeit. $\mathrm{Da}$ Gesetze Interessen regeln, bedeutet ein Urteil über ihre Anwendung Stellungnahme zu einem Interessenkonflikt. Dieses Stellungnehmen ist das Produkt eines sich als Prius des Gesetzes verstehenden Gerechtigkeitsgefühls des Richters, nichts anderes verbergend als dessen klassenbedingtes Sozialideal.

Mit dem neuen Anspruch des Richterstandes korrespondieren durchaus reale Tendenzen. Der andauernde Trend zur Konzentration in der Wirtschaft verlangt, da er fortgesetzt Ungleiches zeugt, Billigkeitsregelungen als Korrektiv oder an Stelle von starr normierten Vertragskonstruktionen. Das Unternehmerinteresse an der Durchlöcherung von Parlamentsgesetzen, sei es die Erzbergersche Steuerreform oder das Betriebsrätegesetz, erfordert adäquate Instrumente der Rechtsprechung. Schließlich duldet der bewaffnete Kompromiß zwischen Kapital und Arbeit als juristisches Ausdrudssmittel nicht präzis normierte Rechtsinstitute, sondern scheint einzig durch Generalklauseln reproduzierbar zu sein, die mit wechselnden Kräfteverhältnissen verschiedene Interpretationsmöglichkeiten bieten.

Doch zeigen sich arbeitsrechtliche Lehre und Rechtsprechung mit dem bloßen Rekurs auf Generalklauseln, die allzu offensichtlich leere, willkürlich füllbare Topoi sind, nicht sehr zufrieden. Generalklauseln werden zur Hebamme eines scheinbar viel Konkreteren, einer Institution, degradiert und damit getarnt. Das zeigt sich etwa in der rechtstheoretischen Diskussion des Arbeitsvertragsrechts und besonders in den Versuchen, das faktische Arbeitsverhältnis in ein System des Arbeitsrechts einzubauen, das wegen seiner kollektiven Qualität mehr sein soll als herkömmliches Zivil-, d. h. Vertragsrecht. Noch hält die herrschende Vertragstheorie formal an der Konzeption des Arbeitsverhältnisses als einem wesentlich schuldrechtlichen Institut fest und behandelt die Regelungen des faktischen Arbeitsverhältnisses als nicht ins System passende Konzessionen an die Wirklichkeit.

Demgegenüber tritt in der Lehre Potthoffs und ähnlich auch in der Molitors ${ }^{8}$ der Vertrag als begründendes Element des Arbeitsverhältnisses immer mehr hinter der Bedeutung der faktischen Einordnung in den Betrieb zurüdk. Für Potthoff ist die hauptsächliche Aufgabe des Arbeitsrechts nicht Regelung des Austausches von Lohn gegen Leistung, sondern $» .$. die Zusammengliederung vieler Menschen zur Arbeirsgemeinschaft des Betriebesk, was ihm eher »Verfassungsrecht als Vertragsrecht bedeutet $^{9}$. Potthoff beruft sich dabei ausdrüdslich auf Otto v. Gierke und dessen personenrechtliche Konzeption, die sich in den $\$ \$ 6$ I7, 6I 8 BGB als sozialkonservatives, deutschrechtliches Korrektiv der romanistischen locatio conductio operarum durchgesetzt hat. Er geht jedoch darüber hinaus, die Unterordnung je einzelner Arbeiter unter die Weisungsbefugnis des Unternehmers festzustellen, indem er das Unternehmen an dessen Stelle setzt. Die Eingliederung in den Organismus des Betriebes schafft überindividuelle Bindungen, ein »Organisationsverhältnis $\alpha$. Damit erscheinen die Wirkungen aus »sozialen Lebensverhältnissen «, die bloße Beschäftigung * wichtiger als das Bestehen eines Vertrages ${ }^{10}$. Der rechtlichen Anerkennung des faktischen Arbeitsverhältnisses und seiner Gleichstellung mit dem vertraglichen steht somit prizipiell nichts mehr im Wege.

Die Konzeptionen Molitors und Potthoffs *... ersetzen den Vertrag durch ein

8 Vgl. Erich Molitor, Arbeitnehmer und Betrieb, Marburg 1929, S. 17 ff.

- Heinz Potthoff, Die Einwirkung der Reichsverfassung auf das Arbeitsrecht, Neuauflage in:

Th. Ramm (Hrsg.), Arbeitsrecht und Politik a.2.O., S. 34 10 Ebenda S. 24. 
einseitiges Diktat. Bisher unterlag die Gestaltung des Arbeitsverhältnisses, wenn auch nur formal, beiden Vertragspartnern. Diese Arr der Gestaltung wird jetzt auf den eventuell vorhandenen Arbeitsvertrag beschränkt, denn dic Einordnung in den Betrieb ist Gewaltverhältnis, Unterwerfung der Person des Arbeitnehmers unter die Herrschaftsgewalt des Arbeitgebers ${ }^{11}$. *

Diese Konzeptionen vermögen das Problem des rechtlichen Schutzes der faktischen Arbeit im Interesse der Arbeiter zu lösen (Anfechtung ex tunc, Regeln über »ungerechtfertigte Bereicherung « sind ausgeschlossen).

Doch die Weimarer Republik kennt ebenso Bestrebungen, die mitrels einer andersartigen Handhabung eines freilich in sehr ähnlicher Weise verselbständigten Betriebsbegriffes den Arbeitern gesetzliche Rechte abzusprechen sich anschicken. Der Betrieb, die »Verdinglichung eines $Z$ wedkes $\alpha$, ist nach Otto Kahn-Freund in der Jurisdiktion des Reichsarbeitsgerichts eine Institution, die als Rechtsquelle sui generis - es gestattet, von den Spannungen zwischen den unter ihr Begriffenen abzusehen. Dieser Begriff, eine nichtssagende $*$ romantische Metapher des Organismus «, dient der Justiz in einem bürgerlichen Rechtsstaat, in dem sie nicht offen Klasseninteressen vertreten darf, weil dessen Existenzgrundlage die Annahme eines prinzipiellen Klassengleichgewichts ist, dazu, allgemein gültige Wertvorstellungen vorzutäuschen ${ }^{12}$. Die Betriebsgemeinschaft wird damit zum Abklatsch der ebenso vage angenommenen und ähnliche Zwecke erfüllenden nationalen Rechtsgemeinschaft auf anderer Ebene. Nur diese Vagheit des Begriffs, die es erlaubt, den $\bowtie$ Betrieb $*$ von seinem Inhaber losgelöst zu denken und zum *höheren Dritten« (Kahn-Freund) gegenüber Unternehmer und Belegschaft zu ernennen, ermöglicht es, den im Betrieb manifesten gesamtgesellschaftlichen Interessenkonflikt zu negieren und scheinbar unparteiisch Recht zu sprechen.

Das RAG verzichter denn auch auf eine ökonomische Definition des Betriebes und bleibt bei einer blassen und tautologischen Bescheibung stehen ${ }^{13}$.

Hinter der "Metapher « des Organismus verbirgt sich die Auffassung, daß seit dem Betriebsrätegesetz von 1920 die persönlichen Bindungen im Betrieb eher mit dem Begriff eines Gemeinschaftsverhältnisses als dem eines der Unterordnung zu beschreiben seien. Nach Sinzheimer bezieht sich die Gemeinschaft im Betriebe .... nicht auf das Vermögen, sondern die Gewalt des Arbeitgebers*. Wäre diese Gemeinschaft aber vermögensrechtlich, so müßte das nicht nur eine "Risikoteilung «, sondern auch "Gewinnteilung « mit sich bringen ${ }^{14}$.

Das RAG judiziert jedoch einseitig auf eine Risikoteilung hin. Den $\ 66$ BRG, demzufolge der Betriebsrat die Betriebsleitung durch *Rat« zu unterstützen und den Betrieb vor "Erschürterungen " zu bewahren hat, nützt es ausgiebig, um feststellen zu können: »Mit der Mitwirkung bei der Leitung des Betriebes Hand in Hand geht ohne weiteres die Mittragung der Gefahren desselben ${ }^{15}$.*

So kann die "Sphärentheorie« konstruiert werden, die \$6 is BGB (Annahmeverzug) ausschließt. So gehen insbesondere soziale Störungen zu Lasten der Arbeiter, was mit der Fiktion ihrer Kontrolle über den Betrieb, mit einer Sozialisierung, die nicht stattgefunden hat, begründet wird. Zudem schickt sich das RAG an, die Arbeiter - nicht nur als Kollektiv der Belegschaft, sondern auch als je einzelne - seiner Konzeption vom Betriebsorganismus zu unterwerfen. Nicht der Individualismus des BGB, sondern die »kollektivistische« Verbunden-

11 Spiros Simitis, a.a.O., S. 327 f.

12 Orto Kahn-Freund, Der Funkrionswandel des Arbeitsrechts, a.a.O., S. 24 I.

13 Vgl. Urteil des RAG vom 8. Februar 1928, Bensheimer Sammiung Bd. 2, S. 7 I und S. 75.

14 Hugo Sinzheimer, Grundzüge des Arbeitsreches, 2. Auflage Jena 1927, S. I 27 u. I=9.

15 Urteil des RAG vom 20. Juni r 928, Bensheimer Sammlung Bd. 3, S. $120 \mathrm{f}$. 
heit soll dem Einzelarbeitsverhältnis seinen eigentlichen Inhalt geben. In einem besonders extremen Urteil richtet sich dieser Grundsatz gegen einen Betriebsratsvorsitzenden, dessen Entlassung das RAG mit der Begründung bestätigt, seine Ablehnung von Mehrarbeit über den gesetzlichen 8-Stundentag hinaus müsse als »beharrliche Arbeitsverweigerung " gelten. Der Mann, der nichr »Partei, sondern Berater der Betriebsleitung sei«, habe sich "einseitig* und nicht "pflichtgemäß verhalten ${ }^{16}$. Allgemein wird die exakte Bestimmtheit der Leistungspflicht, die ein Wesensmerkmal des Schuldverhältnisses ist, immer mehr ersetzt durch eine "generelle Unterordnung* (Kahn-Freund) unter den Willen des Vertragsgegners, d. h. das Arbeitsverhältnis wird ähnlich einem Beamtenverhältnis konstruiert.

Der Treuepflicht des Arbeitnehmers entspricht die Fürsorgepflicht des Dienstberechtigten. Hierher gehören die Bindung an die Stelle, die sich besonders in der Ausdehnung des Kündigungsschutzes für Angestellte ausdrückt, und die Bejahung von Zwangspensionskassen durch das RAG. Hinzu kommt eine gehörige Portion Mitleid, das der kleinbürgerlichen Komponente des richterlichen Sozialideals entstammt und sich besonders zugunsten kranker Arbeitnehmer auswirkt etwa in der erschwerten Kündigung Schwerbeschädigter. Otto Kahn-Freund sagt von dieser Konzeption, daß sie auf eine "disziplinäre Harmonie hinziele und von "... . einer auf der einen Seite romantisch-militärischen, auf der anderen Seite ausgesprochen kleinbürgerlichen Einstellung ${ }^{17}$ " ausgehe, was mit den sozialen Leitvorstellungen des Arbeitsrechts im Faschismus übereinstimme. Nach einer Analyse der italienischen Arbeitsgesetze von 1926 und 1927 konstatiert er für das faschistische Sozialideal eine eigentümliche Mischung von liberalen, kollektivistischen und sozialkonservativen Komponenten.

Liberal ist die Freiheit vor Eingriffen des Staates in die Produktionslenkung, sozialkonservativ die individuell eingreifende staacliche Fürsorge und spezifisch faschistisch die Bindung kollektivistischer Körperschaften an einen immerwährenden Wirtschaftsfrieden aus nationalem Interesse ${ }^{18}$. Auch das RAG postuliert die Idee des "Wirtschaftsfriedens", die sich auf Arbeitskämpfe regulierend auswirken soll, und maßt sich sogar an, Tarifverträge an Treu und Glauben zu binden - dabei mit Selbstverständlichkeit die Auslegung beanspruchend. Dem entsprechen terminologische Veränderungen, die den statischen "Stand" an die Stelle der dynamischen »Klasse« setzen und damit Kooperation statt Kampf suggerieren.

Nicht sehr entfernt sind solche Bestrebungen von den Angriffen der Unternehmer und der Harzburger Front auf die Unabdingbarkeit des Tarifvertrages, womit in der Endphase der Weimarer Republik eine der Haupterrungenschaften dieses Staates bedroht wird. Dem Kampf gegen den Tarifvertrag entsprechen die Versuche, die Betriebsräte gegen die Gewerkschaften auszuspielen.

Dennoch ist das Arbeitsrecht, das "Prunkstück der Republik « (E. Fraenkel), wesentliches Bindeglied zwischen Arbeiterschaft und Staat. Die Arbeitsgerichte, an denen Arbeitervertreter neben Unternehmervertretern als Repräsentanten ihres Verbandes an der Rechtsfindung teilnehmen, stellen für das Proletariat in ihrer Wirksamkeit sichtbare Institutionen des Interessenausgleichs dar. Sie vermitteln den Eindruck direkten Einflusses, den die Arbeiter gegenüber der konservativen Bürokratie und den Weimarer Koalitionsregierungen längst verloren haben. Das

\footnotetext{
16 Urteil des RAG vom 29. Mai r929, Bensheimer Sammlung Bd. 6, S. 335.

17 Otto Kahn-Freund, Das soziale Ideal des Reidsarbeitsgeridhts, in: Th. Ramm, a.a.O., S. I92.

$18 \mathrm{Vgl}$. ebenda S. IS2.
} 
Engagement des Proletariats fürs Arbeitsrecht, Ausdrudk des Abgleitens der Revolution ins sozialpolitisch-arbeitsrechtliche Fahrwasser, ist als Folge der mißlungenen Umwandlung der Gesellschaft eine Arr Ersatzbefriedigung, ein Zeichen der Schwäche.

III.

In der Zeit des Nationalsozialismus ${ }^{10}$ sind staatliche Eingriffe in frühere Grundfreiheiten allgegenwärtig. Mir der fortgeschritrenen Zerstörung seines sozialen Substrats, des Konkurrenzkapitalismus, verschwindet der Vertrag und damit die Geschlossenheit der Privatrechtsordnung.

"Das neue Konnexinstitut zum Schutz des Eigentums wird der unmittelbare Befehl des Staates, der Verwaltungsakt, der in direkter Weise Interessen des Monopolisten schützt und die alten Garantien beschränkt oder beseitigt ${ }^{20}$. «

Auch der Kollektivvertrag zwischen den Koalitionen von Arbeit und Kapital wird abgeschaff. Vom Reichstreuhänder der Arbeit verhängte staatliche Tarifordnungen treten an die Stelle des Tarifvertrages. Damit steht institutionell der Stabilisierung des Lohnsrops auch in der Phase der Konjunktur nichts mehr im Wege, denn ständig wachsende Rüstungsinvestitionen fordern Konsumverzicht.

Der Befehl verwirklicht sich durch das Mittel der Generalklauseln hindurch, die damit die Funktion erhalten, in einer Ubergangszeit bis zur Existenz nationalsozialistischer Kodifikationen, die freilich auch nicht vor dezisionistischen Eingriffen geschützt sind, das neue »Recht« gegen das herkömmliche durchzusetzen.

Richter werden zu "Polizisten « des Führers (Franz Neumann), Gesetze haben nur als virtuelle Führerbefehle Geltung und unterliegen nicht mehr dem strikten Verbot der Rückwirkung. In diesem »Rechtsstaat" neuer Art, gilt die Gleichheir der Menschen vor dem Gesetz nicht mehr, da die Rechtsperson als Eckpfeiler des formal-rationalen Systems durch »die konkrete Persönlichkeit" " als Glied einer Gemeinschaft abgelöst wird.

Die Konstruktion der »konkreten Persönlichkeit« gehört dem Bereich der wesentlich institutionalistisch geprägten Rechtsideologie des Nationalsozialismus an, während seine eigentliche juristische Theorie der Dezisionismus ist. Der Institutionalismus, das »konkrete Ordnungs- und Gemeinschaftsdenken "Carl Schmitts"2, "gebärdet sich als progressive, als enthüllende Theorie dadurch, daß er dem Begriff der Person den Kampf ansagt und ihn durch den der Institution ersetzt, die nicht wie die liberalistische Rechtsperson Differenzierungen verhüllt23." Doch kennt er keine anderen Begriffe zur Analyse von Strukturen als die der Differenzierung und der Gliederung. Er hypostasiert "Gemeinschaft" überall da, wo in Wahrheit extreme Antagonismen bestehen. Hinter der *Volksgemeinschaft « mit ihren organischen Gliederungen verschwindet die Herrschaft einer parasitären Gruppe völlig und die »Betriebsgemeinschaft" eskamotiert auf einer anderen Ebene - in derselben tarnenden Funktion den Unternehmer

19 Die gängigen Lehrbücher etwa des Arbeitsrechts behandeln diese Phase eigentümlich lakonisch. -0 F. Neumann, Der Funktionswandel ... a.a.O., S. 67.

21 Vgl. Karl Larenz, Rechrsperson und subjektives Recht, Tübingen r935, S. 9.

29 Vgl. Carl Schmitr, Uber die drei Arten des rechtswissenschaftichen Denkens, Hamburg 1934

${ }^{23}$ F. Neumann, Der Funktionswandel ... a.3.O., S. 69. 
aus der arbeitsrechtlichen Theorie, die damit als Werkzeug wirtschaftlicher Macht

sich entpuppt.

Laut Gesetz zur Ordnung der nationalen Arbeit« setze der Unternehmer, der regelmäßig auch der Führer des Betriebes ist, Betriebsordnungen und Betriebsbedingungen fest.

Die Konstruktion eines »Vertrauensrates« bringt die Jurisdiktion des RAG zum Betriebsrätegesetz auf ihren Begriff: Dieses Gebilde soll kaum mehr als ogegenseitiges Vertrauen vertiefen ( $\$ 6$ des Gesetzes) und sieht besonders bei Störung des Betriebsfriedens durch die Gefolgschaft, sowie Eingriffen der nicht frei gewählten Vertrauensleute in die Betriebsführung "Ehrengerichts «verfahren vor $(\$ 36)$. Spannungen werden hinwegdekretiert. So ist der Inhalt der Institution, die vorgeblich Rechtsquelle sein soll, auf den dezisionistischen Befehl reduzierbar.

Wie läßt sich aber die Fortexistenz der arbeitsrechtlichen Vertragstheorie erklären, während der Nationalsozialismus die seiner institutionalistischen Ideologie entsprechende, betriebsbezogene und vertragsfeindliche Eingliederungstheorie geschaffen hat? Die Jurisdiktior des RAG nach 1933 zu Streitigkeiten aus Einzelarbeitsverhältnissen bezieht sich immer dann, wenn es gilt, ein schuldrechtliches Prinzip nicht anzuwenden, auf personenrechtliche Konstruktionen oder auf den Begriff der Betriebsgemeinschaft ${ }^{24}$. Diese Konzessionen neutralisieren die Vertragstheorie und belassen dem Vertrag - nach Abschaffung des schützenden Tarifvertrags - nur noch die Aufgabe, die Entstehung von Arbeitsverhältnissen rechtlich übersichtlicher zu machen. Damit bleibt er nichts als ein rationelles Werkzeug der Kontrolle des Arbeitsmarktes. Das reicht aus, einigen Juristen die Illusion zu belassen, an der vorgegebenen gesetzlichen Regelung festzuhalten, d. h. dort Rechtsdogmatik zu betreiben, wo alle Dogmen durch eine neue Rechtspolitik längst in Frage gestellt sind.

Dieses Nebeneinander von juristischen Theorien paßt sich durchaus in die politische Struktur des Nationalsozialismus ein, die über dem Wirrwarr der Instanzen, über der ständig vertagten Austragung von Interessenkonflikten, der feindlichen Parallelität der Verwaltungswege - etwa des Staates und der Partei - nur einen Fixpunkt kennt: den Führerbefehl. Gerade die Kompetenzunsicherheit der Exekutivorgane, die jeweils durch konkurrierende Institutionen ersetzbar scheinen, ermöglicht die Allgegenwart des Eingriffs der Zentrale. Das ist eine wesentliche Vorbedingung für die Militarisierung der gesamten Gesellschaft, zu der auch - und vor allem - die Entmactinng der Arbeiterschaft gehört.

IV.

Die mit dem Grundgesetz der Bundesrepublik wiedereingesetzte Gewerbe- und damit auch Vertragsfreiheit bringen die arbeitsrechtliche Vertragstheorie zu neuer Würde. Ihre scheinbare Dominanz ist jedoch mit Zugeständnissen an die Eingliederungstheorie erkauft, deren wichtigster Vertreter neben Siebert, Nikisch, nun auf einmal das betriebsbezogene Gemeinschaftsverhältnis nur als eine "spätere Phase des schon mit dem Inkrafttreten des Arbeirsvertrages beginnenden Arbeitsverhältnisses ${ }^{25}$ « ansehen will.

Als Gegengabe interpretieren Hueck und Nipperdey, wichtige Vertreter der Ver-

24 Vgl. Spiros Simitis, a.a.O., S. 365 ff.

23 A. Nikisch, Arbeitssecht, 3. Auflage, Bd. r, Tübingen I96r, S. VIII. 
tragstheorie, den Arbeitsvertrag als personenrechclich und gemeinschaftsbegründend, sehen ihn nicht als romanistische und gemeinrechtliche Dienstmiete sondern eher als "Gesellschaftsvertrag . Widerwillig heißr es: "Doch verlangt die Gesetzestreue, da das BGB den Arbeitsvertrag zu den Dienstverträgen rechnet und diese im Schuldrecht regelt, daß von den Normen des Schuldrechts als maßgebend ausgegangen und von ihnen nur dann abgewichen wird, wenn die Notwendigkeit der Abweichung wirklich dargetan ist ${ }^{2 \mathrm{e}}$. "Eine solche Notwendigkeit besteht z. B. in der Anerkennung des faktischen Arbeitsverhältnisses, für das in praxi Vertrags- und Eingliederungstheorie zu identischen Ergebnissen kommen. Diese Harmonie formuliert sich bei Hueck und Nipperdey so: "Der Arbeitnehmer ist Mitglied der Betriebsgemeinschaft mit allen sich daraus ergebenden Folgen gewesen. Es ist aber auch zwischen ihm und dem Arbeitgeber ein Gemeinschaftsverhältnis begründet worden, das zu persönlichen Beziehungen geführt hat, die nicht mehr aus der Welt geschafft werden können. Eine rückwirkende Negierung dieser Rechtsbeziehungen und ihr Ersatz durch bloße Bereicherungsund gegebenenfalls Schadenersatzansprüche führt zu wenig erfreulichen Folgen $^{27}$. Innerbetriebliche Herrschaft wird so zur Quelle des Schutzes faktischer Arbeit, nicht aber die gesellschaftliche Widersprüche erfassende Einsicht in die Lage der von der Kontrolle der Produktionsmittel ausgeschlossenen Verkäufer der eigenen Arbeitskraft.

Von bemerkenswerter Konstanz sind bestimmte arbeitsrechtliche Denkfiguren, die in ihrer Stereorypie feste Topoi bilden: Wie in Weimar haben sich auch in der Bundesrepublik Streiks an richterlich interpretierten Generalklauseln zu messen. War es damals "nur" $\ 826$ BGB (sittenwidrige vorsätzliche Schädigung) ist es heute $\$ 823$ I BGB (schuldhafte Verletzung ausschließlicher Rechte), der, weil er bereits Fahrlässigkeit als schuldhaft ansieht, haftungsverschärfend wirkt.

Diese Restriktion des Streikrechts gelingt Nipperdey, indem er durch sein Zeitungsstreikgutachten und über die Rechtsprechung des Bundesarbeitsgerichtes dessen erster Präsident er war - die Sozialadäquanztheorie etabliert. Streiks, als Eingriffe in das Recht "am eingerichteren und ausgeübten Gewerbebetrieb « erfahren eine Neuwertung als prinzipiell unerlaubte Handlung, die sich erst als sozialadäquat zu rechtfertigen hat. Die richterlich fixierten Bedingungen der Sozialadäquanz sind beliebig und vor allem beliebig zu vermehren. Einer "Salamitaktik " (Thilo Ramm) gegen das Streikrecht ist der Weg eröffnet. Vorläufiger Endpunkt dieser Bestrebungen ist der Vorschlag Bullas, die Sozialadäquanz an die Gutachten des Sachverständigenrats zu binden ${ }^{23}$.

Wie in Weimar wird heute behauptet, der "Betriebsratsgedanke" solle bewirken, daß der Arbeitnehmer den $\bowtie$ Betrieb als den seinen empfindet, zu ihm ein persönliches Verhältnis gewinnt, ein stärkeres Interesse an seinem Gedeihen und damit auch an seiner eigenen dem Betrieb gewidmeren Arbeit erlangt ${ }^{29}$. So leicht überwinden Juristen die Entfremdung der Arbeit! Und Dietz wagt es in seinem Kommentar zum Betriebsverfassungsgesetz, durchaus affirmativ auf das AOG zu verweisen, auch zu dessen Kommentatoren er bereits gehörte ${ }^{30}$.

Nach dem Streik der IG Metall in Bayern 1954 werden aktiv am Streik beteiligte Betriebsratsmitglieder, wegen Gefährdung ihrer besonderen Verbundenheit mit dem Betrieb entlassen, in weitherziger Auslegung des $\ 49$ Betr.Verf.G.

26 Hued-Nipperdey, Grundriß des Arbeirsrechrs, 3. Auflage, Berlin/Frankfurt 1965, S. 43 f.

27 Ebenda S. 55 .

28 Vgl. Bulla, Fesrschrift für Nipperdey, München und Beriin 1969, Bd. II, S. 96 ff.

29 Hueck-Nipperdey, a.a.O., S. 22 .

$30 \mathrm{Vgl}$. H. Lenz, Die unbehagliche Nähe der Koalitionsgarantie zum Sozialstaat, in: Festschrift für A bendroth, Neuwied 1968, S. 231, Fußnore 102. 
Diese Konstanz mag zu vorschnellen Personalisierungen verleiten - etwa nach dem Muster: Was wäre, hätte es Hans Carl Nipperdey nie gegeben? Ebenso lenkt die Gerontokratie der Hueck, Nipperdey, Nikisch, Siebert, Molitor, Dietz et alii - zum Teil bereits seit Weimar - von der Auswechselbarkeit dieser Personen $a b$. Ohne soziale Veränderungen zu provozieren scheinen sie ersetzbar durch einen Juristenstand, der sich beinah wie ehedem rekrutiert. Die Ergebnisse der Untersuchung W. Richters über „Die Richter der Oberlandesgerichte der Bundesrepublik ${ }^{31}$ * dürften annähernd auch für andere Teile der beamteten Justiz gelten:

»r. Wie andere akademische Berufsgruppen rekrutieren sich die Richter vorwiegend, nämlich zu fast zwei Dritteln, aus den oberen fünf Prozent der Statushierarchie.

2. Wie andere akademische Berufsgruppen - insbesondere die Lehrer - stammen über die Hälfte der Richter aus Beamtenfamilien, wobei höhere Beamte einerseits, Beamte des gehobenen, mittleren und einfachen Dienstes andererseits sich etwa die Waage halten.

3. Im Gegensatz zu anderen Eliten stammt ein unverhältnismäßig hoher Prozentsatz der Richter, nämlich ein Viertel, aus Juristenfamilien.

4. Bei Richtern, wie bei Juristen allgemein, ist der Anteil derer, die sich aus Arbeiterfamilien rekrutieren, besonders gering ${ }^{32}$.

Daran anknüpfend spricht Dahrendorf von »sozialer Immobilität" und nkonservativer Haltung ". Dabei setzt er konservativ mit der Fixierung ans »geltende Recht " gleich. ${ }^{33}$. Von der Orientierung an der gültigen gesellschaftlichen Macht notfalls auch gegen Normen - weiß er nichrs. So vermag das mechanische $\mathrm{Zu}$ sammenbringen einer herkömmlichen Vorstellung des "Konservativen " mit den Befunden einer spezifischen Rekrutierungsweise, eine Analyse richterlicher Rechtspolitik nicht zu ersetzen.

$V$.

Die bürgerlichen Arbeitsrechtler erheben sich zu Interpreten und Vertvaltern eines gesellschaftlichen Kompromisses. Diesen »ideologisieren « sie - »unversöhnte Interessenlagen « ignorierend - und setzen ihn somit »zu einem Statusvertrag innerhalb des fingierten Rahmens einer im Prinzip konfliktfreien Ordnung her$a b^{34}$.

Mit dem Kompromiß nicht vernichtete gesamtgesellschaftliche Widersprüche sollen zugedeckt werden, indem etwa das Streikrecht an ein elastisch handhabbares „Ordnungsprinzip « gebunden, indem das Individualarbeitsverhältnis seiner vertraglichen Normierung immer mehr entkleidet wird, die sich mit einem konfliktfreien Betriebsgemeinschaftsbegriff zwangsversöhnen lassen muß oder Umgestaltung durch personenrechtliche Konzeptionen erfährt - damit kalkulierbaren Leistungsaustausch durch persönliche Verbundenheit und den Arbeitern aufgedrängte menschliche Wärme ersetzend.

31 W. Richter, Die Richter der Oberlandesgerichte der Bundesrepublik, in: Hamburger Jahrbuch für Wirtschafts- und Gesellschaftspolitik, 5. Jahrgang, Tübingen r960, S. $24 \mathrm{r}$ ff. Vgl. auch J. Feest, Die Bundesrichter. Herkunft, Karriere u. Auswahl der juristischen Elite, in: W. Zapf (Hrsg.) Beiträge zur Analyse der deurschen Oberschicht, München 1965.

32 R. Dahrendorf, Deursche Richter, in: Gesellschaft und Freiheit, München r962, S. 189.

33 Ebenda, S. 193

34 Jürgen Habermas, Struksurwandel der Offentichkeit, Neuwied r965, S. 2r8, FuBnote 49. 
*Das früher rein vertragliche Arbeitsverhältnis ist nun Stelle geworden, wie das Eigentum Anstalt. Man hat eine Stelle inne mit festem Rechts- und Pflichtenkreis, und diese Stelle ist ein ähnliches Rechtsinstitut geworden wie das Lehen der Feudaizeit. Die Stelle umfaßt den Anspruch auf gebührenden Lohn (Tarif oder Pragmatik), die Pflicht bestimmter Beitragsleistungen (Gewerkschaft, Versicherungsinstitute), das Recht auf bestimmte Versorgungsgenüsse (Krankheit, Unfall, Alter, Tod) und endlich bestimmte Sicherungen gegen Stellenverlust... ${ }^{35}$. " Das bedeutet die Umkehrung der Bewegung from status to contract. Die Gültigkeit dieser These wird jedoch eingeschränkt durch die Erkenntnis, daß die Ahnlichkeit von Lehen und Stelle ihre Grenzen hat: Erbliche oder unkündbare Stellen etwa sind für einen industriellen Großberrieb - trorz deutschen "Kruppianertums - wohl kaum auch nur rechtlich konzipiert worden. Bereits die Sicherung gegen "Stellenverlust « zeigt durchaus schwerwiegende Lücken: Die Kündigung aus »wichtigem Grunde " durchbricht den gesetzlichen Kündigungsschutz. Zudem sind Angestellte hier gegenüber Arbeitern privilegiert. Das verweist auf die soziale Funktion der Sicherung der Stelle - des Status: die Integration der abhängigen Arbeit in die spätkapitalistische Gesellschaft. DaB hierbei einer Gruppe der Arbeitnehmer, den Angestellten, der Vortritt gelassen wird, macht durch Teilung den Block des Proletariats nur eher manipulierbar. Die Unterscheidung zwischen Arbeitern und Angestellten ist dann auch recht willkürlich, »eine Legaldefinition fehlt ${ }^{36} \ll$.

Das im Individualarbeitsvertrag enthaltene Versprechen, frei die Arbeitsbedingungen aushandeln zu können, wird nicht eingelöst - auch mit der Existenz und dem Schutz des Tarifvertrages nicht. Der Tarifyertrag ist Produkt jenes Kompromisses, in den die am System orientierte, Konjunkturschwankungen ausgelieferte Macht der Arbeiterkoalitionen mit eingeht. Damit erscheint er gleichzeitig als Ausdruck der Schwäche dieser Koalitionen, die um seinetwillen und im $\mathrm{Na}$ men der Ordnung verzichtet haben, für die Verwirklichung eines Gesamtinteresses der Gesellschaft zu kämpfen. Diese Schwäche läßt Bestrebungen Raum, den Arbeitsvertrag mittels ideologischer Verbrämungen betrieblicher Herrschaft zu modifzieren und dem Begriff der Stelle anzunähern, um so mehr als die Arbeiter - der Alternative einer Umgestaltung der Produktionsverhältnisse beraubt - die von dieser Herrschaft gewährte Fürsorge durchaus benötigen.

Diese Bewegung vom Vertrag zur »Stelle« ist durchs Arbeitsrecht vermittelt, das dafür von seinen bürgerlichen Vertretern mit einem hohen Anspruch versehen wird. Danach hat es die Aufgabe »der Uberwindung der sozialen Gegensätze, der Lösung der sozialen Frage, der Herstellung und Aufrechrerhaltung des sozialen Friedens und damit der Herbeiführung einer echten Volksgemeinschaft ${ }^{37}{ }^{4}$. Dabei »ist es ... nicht zulässig, alle Bestimmungen des Arbeitsrechts nur zugunsten der Arbeitnehmer anzuwenden ${ }^{38}{ }_{\alpha}$.

Thilo Ramm hat, wie früher Neumann dem RAG, dem Bundesarbeitsgericht vorgeworfen, daß jener Anspruch die Richter - um eigener sozialer Leitbilder willen - veranlaBt habe, sich über die Bindung ans Gesetz zu erheben. Rechtspolitik verdränge Rechtsdogmatik. Das wirke sich gegen die Interessen der zu schützenden Arbeiter, vor allem gegen die Autonomie ihrer kollektiven Vertretungen, aus. Neumann und Ramm halten deswegen den Satz von der Unab-

95 Karl Renner, Die Rechrsinstiture des Privatrechts und ihre soziale Funktion, Stutrgart 1969, S. 102.

${ }^{38}$ Hans $G$. Joadhim, Erziehung zum Arbeitsrecht, in: Recht und Politik, Heft r, Berlin 1966, S. 4

${ }^{37}$ Hueck-Nipperdey, a.a.O., S. 4 .

38 Ebenda, S. 21. 
hängigkeit des Richters - wenn er gegen das Gesetz gerichtet wird - für überprüfbar ${ }^{39}$. Konkrete Vorstellungen über Sanktionen gegen allzu »königliche« Richter entwidkein sie jedoch nicht.

Die Anwendung von Generalklauseln im Arbeitsrecht impliziert nicht nur die Konstruktion vager Rechtsbegriffe im Interesse richterlicher Rechtspolitik, sie bedeutet auch, daß eine unübersichtliche Vielzahl von Einzelfallentscheidungen an die Stelle fester Normen tritt und so Rechtsunsicherheit erzeugt wird. Die Kodifikation des Arbeitsrechts mißlang schon in der Weimarer Republik. Der 1919 eingesetzte Arbeitsrechtsausschuß stellte mit der Inflation aus "finanziellen Gründen « seine Tätigkeit ein. In der Bundesrepublik fehlt ebenfalls eine Gesamtkodifikation. Hans G. Joachim spricht vom "Arbeitsrecht als Geheimwissenschaft" und in diesem Zusammenhang von einem "Scheinfrieden in Rechtsnot" aus Unkenntnis der Rechtslage ${ }^{t 0}$. Das aus dem Kompromißcharakter des Arbeitsrechts sich ergebende Erfordernis elastischer Normen wirkt sich so auch unter dem Aspekt der Rechtssicherheit vor allem gegen die Arbeiter aus, denn ihnen steht - anders als den Unternehmern - professioneller Rechtsbeistand wohl kaum ständig zur Verfügung..

Treiben Richter Rechtspolitik, wird die Rolle des Parlaments als Medium der Verwandlung gesellschaftlicher Interessen in Recht eingeschränkt. Recht - ein Ausdruck der Vermittlung von Staat und Gesellschaft - wird damit durch die in der Sphäre der Gesellschaft selbst wirkenden Repräsentanten des Staates geschaffen - nicht mehr in einer von ihr deutlich sich abhebenden Institution. So dringt die staatliche Rechtsschöpfung in die Gesellschaft ein. Dem Einfluß gesellschaftlicher Macht aber sind die Träger dieser Rechtsschöpfung, die Richter, durch ihren sozialen Ort unvermitteiter und unkontrollierbarer ausgesetzt als je eine parlamentarische Regierung es sein kann.

In der neuen Rolle der Richter zeigt sich ein weiterer Aspekt der »Refeudalisierung* der Gesellschaft, die Jürgen Habermas mit dem Begriff der »Verschränkung von öffentlichem und privatem Bereich"1 " beschreibt. Daß auch die Ursprünge des Arbeitsrechts - wie der staatliche Arbeitsschutz und die staatliche Sanktionierung des Tarifsetzungsaktes gesellschaftlicher Institutionen - diesem Begriff entsprechen, ist eindeurig.

Otto Kahn-Freunds Hinweis auf die Verwandtschaft des sozialen Ideals des Reichsarbeitsgerichts mit faschistischen Ordnungsvorstellungen ist auch auf die Rechtsprechung des Bundesarbeitsgerichts übertragbar. Er verliert jedoch an unmittelbarer Schärfe, weil sich wegen der fortgeschrittenen Integration des Proletariats ins System des organisierten Kapitalismus ein staatlich verordneter oder empfohlener Wirtschaftsfrieden - etwa nach dem Muster der »konzertierten Aktion - nun offenbar auch ohne die typisch faschistische Abschaffung der formalen Garantie persönlicher und politischer Freiheitsrechte liberaler Herkunft realisieren läßt. Dabei kann die Existenz von Notstandsgesetzen, sei es nur, um Argumenten Nachdruck zu verleihen, durchaus willkommen sein.

30 Vgl. Franz Neumann, Die politische und soziale Bedeutung der arbeitsgerichtichen Rechrsprechung, Berlin s929, S. 16 if.

Vgl. Thilo Ramm, Die Rechtsprechung des Bundesarbeitsgericits in: JZ I 964, S. $582 \mathrm{ff}$.

10 Joachim, a.a.O., S. 4 u. S. 7 .

4 J. Habermas, a.a.O., S. $25 \mathrm{I}$. 